\title{
Sonstiges
}

\section{Die «Fadenspule» in Zürich - ein psychoanalytisch orientierter Begegnungsraum für Kleinkinder und ihre Eltern}

\author{
Dagmar Ambass (Zürich)
}

In Zürich wird zurzeit an der Realisierung eines Begegnungsortes, angelehnt an das Konzept der «Maison Verte» ${ }^{1}$, gearbeitet. Das Angebot richtet sich an Kinder von null bis vier Jahren und ihre Eltern oder andere nahe Bezugspersonen. In der so genannten «Fadenspule» sind stets zwei bis drei Personen mit einem Bezug zur Psychoanalyse und Erfahrung im Frühbereich anwesend. Diese Begleitenden haben ein offenes Ohr für die Anliegen der Eltern und sind sensibel für die frühen Ausdrucksformen und Kommunikationsmöglichkeiten der Kinder. Die Fadenspule ermöglicht den Kindern, erste soziale Erfahrungen ausserhalb des familiären Umfelds.

Wir - das sind Antje Brüning, Helen Stierlin, Oezlem Schneider, Ursula Scheuchzer und ich - bieten den Begegnungsort seit April 2009 regelmässig an.

Zur Entstehung und zum Konzept der «Maison Verte» Paris und anderer «lieux d'accueil» ${ }^{2}$

Die «Maison Verte» öffnete am 6. Januar 1979 in Paris ihre Türen. Ihren Namen erhielt sie aus Kindermund: Die Kleinen machten aus der «Maison Ouverte» eine «Maison Verte».

Es war ein Anliegen der Gründer und Gründerinnen, Familien einen Raum für den sozialen Austausch zur Verfügung zu stellen und ungünstigen Entwicklungen in der Eltern-Kind-Beziehung zu einem frühen Zeitpunkt vorzubeugen. Die Gründung fällt in die Zeit der 70er Jahre, wo aufgrund von Migrationen die Einbindung im erweiterten Familienverband zunehmend wegfiel, und wo viele Mütter mit ihren Kindern in den eigenen vierWänden ziemlich isoliert waren. Die 
ersten Erfahrungen zeigten, dass dieWirkung der Maison Verte für die Kinder und ihre Bezugspersonen unter anderem in einer Übertragung auf den Ort geschieht. Das heisst, ein Arbeitsbündnis entwickelt sich nicht nur in der Beziehung zu den einzelnen Personen sondern auch gegenüber dem Ort.

Wichtig war den Gründern und Gründerinnen die Funktion der Maison Verte als «lien social» (soziale Bindung). Familien, die isoliert leben, finden in der Maison Verte einen Ort, wo sie Kontakte knüpfen und wo Beziehungen entstehen können.

Dieses Modell ist an vielen anderen Orten in Frankreich und in weiteren Ländern aufgenommen worden, wobei jeder dieser Orte seine eigene Identität finden muss. Einer davon ist der «CerfVolant» in Genf und es gibt weitere in der französischsprachigen Schweiz. In St. Petersburg beispielsweise, wo in der Zeit des Kalten Krieges die Rezeption der Psychoanalyse zum Stillstand gekommen war, entstand im Zuge der Öffnung aufgrund von persönlichen Kontakten eine Einrichtung nach dem Modell der Maison Verte. Ihre Schwierigkeit bestand darin, dass in Russland keinerlei Gesprächskultur, was die eigene Geschichte und Befindlichkeit anbelangt, existierte. Die Frage, die die Mitarbeitenden in der Anfangsphase beschäftigte, war: Wie kann man die Menschen überhaupt dazu bewegen, über sich zu sprechen?

Für uns in Zürich ist ein zentrales Thema die Integration von Migranten und Migrantinnen. Wie bringt man sie dazu, die bestehenden Angebote und Einrichtungen aufzusuchen? Dies ist eine der Aufgaben, die wir uns stellen.

\section{Zur Entstehung der «Fadenspule» ${ }^{3}$}

Als Tätige im Kleinkindbereich und Interessierte an der Lacanschen Psychoanalyse und an Möglichkeiten, mit den Mitteln der Psychoanalyse im sozialen Feld zu arbeiten, fragten wir uns, warum das bewährte Projekt bisher im deutschsprachigen Raum noch keinen Platz gefunden hatte, und beschlossen, uns für die Entstehung eines Begegnungsortes in Zürich einzusetzen. Der Verein für Psychoanalytische Sozialarbeit (vpsz) stellte uns für die ersten Entwicklungsschritte sein Dach zur Verfügung. Wir begannen zu planen, diskutieren und ein Konzept zu entwerfen. Dieses präsentierten wir im Kleinkindbereich tätigen Personen. Die Fachpersonen ermutigten uns in unserem Vorhaben, indem sie uns versicherten, dass ein niederschwelliger Ort mit einem offenen Angebot und gleichzeitig klaren Strukturen vielen Eltern eine wichtige Orientierung bieten würde. Konkret unterstützten uns Mitarbeiterinnen des Marie-Meierhofer-Instituts (Dr. Heidi Simoni und Sabine Brunner) bei der Konzeption des Pilotprojekts und Hansjörg Haas, Leiter des Kinderhauses Entlisberg (einer städtischen Einrichtung mit ver- 
schiedenen Angeboten wie einem Kinderheim, Krippen, Spielgruppen), durch das Angebot, die Räumlichkeiten des Kinderhauses für ein Pilotprojekt zu nutzen. Das Lacan Seminar Zürich bekundete seine Solidarität mit dem Projekt, indem es gemeinsam mit dem vpsz die Kosten zum Erstellen von Werbematerial und Broschüren übernahm. In der «Maison Verte» konnten wir anfangs letzten Jahres für einen Nachmittag am Betrieb teilnehmen und mit den anwesenden Mitarbeitenden diskutieren.

In Zürich gab es bereits mehrere Anläufe, ein Angebot in Anlehnung an die «Maison Verte» einzuführen. Ein Beispiel dafür ist «die Oase», gegründet 1987 von der Psychoanalytikerin Eva Burkard in Zusammenarbeit mit drei weiteren engagierten Frauen, die als Angebot des Jugendsekretariats Glattal geführt wurde (Saller und Würgler 1992). Auch wenn eine dauerhafte Institutionalisierung bisher nicht gelungen ist, scheint der Ruf danach doch immer wieder laut zu werden. Ausserdem inspirierte uns die Zürcher Kinder- und Jugendpsychiaterin und Psychoanalytikerin Fernanda Pedrina. Ihre Forschungstätigkeit über die Arbeit mit Müttern und Babys in Gruppen brachte sie zu der Auffassung, dass Kleinkinder bereits im Säuglingsalter eine innere Repräsentanz für die Gruppe ausbilden können (Pedrina 2010: 28-52). Für die Kinder kann es bereichernd sein, wenn ihnen ein Ort für Interaktionen in der Gruppe zurVerfügung steht, wo sie mit dem Loslösen experimentieren können. Aber auch die Eltern brauchen einen Ort, wo sie Worte finden können für das, was mit ihnen beim Eintritt in die Elternrolle geschieht. Vielleicht könnte man die «Fadenspule» in Anlehnung an Winnicotts (2010) Begriff des Übergangsobjekts als Übergangsraum von der Familie in die Gesellschaft und die damit verbundenen Rollen auffassen.

Ähnlich wie die «Maison Verte» ist die «Fadenspule» ein einladender Ort, der einerseits einen Quartierbezug aufweist und andererseits zentral gelegen ist, so dass er auch für weiter entfernt lebende Familien erreichbar ist. Sie ist an zwei Vormittagen geöffnet und kann ohne Voranmeldung und ohne dass ein spezifisches Anliegen formuliert werden muss, besucht werden. Besucher und Besucherinnen schätzen die grosszügigen Räumlichkeiten und das interessante Spielmaterial, das sich an den Konzepten von Emmi Pikler (2001) orientiert. DerVorname und das Alter des Kindes, sowie die Rolle der Begleitperson (Mutter, Grossvater, Erzieherin etc.) werden bei der Ankunft auf einer Tafel notiert. Wichtig ist, dass das Kind von seiner Bezugsperson in der «Fadenspule» nicht allein gelassen wird. Das Kind kann sich durch die Anwesenheit der Mutter genügend sicher fühlen, um erste Erfahrungen beim Aufnehmen von Kontakten zu sammeln. So lernt es ein Umfeld kennen, das über die Familie hinausgeht. Aufgrund ihrer Erfahrung als Kinderanalytikerin 
erkannte Dolto, dass kleine Kinder beispielsweise bei einem frühen Krippeneintritt oftmals einer zu abrupten Trennung von der engsten Bezugsperson ausgesetzt sind und dies in einem Augenblick, wo das Identitätsgefühl des Kindes noch mit dem seiner Mutter eng verbunden ist. Dolto (1995) zeigte, dass eine nicht vorbereitete Trennung in den ersten beiden Lebensjahren vom Kleinkind traumatisch erlebt werden und zur Entwicklung von Neurosen führen kann.

\section{Warum ein weiteres Angebot in Zürich?}

Obwohl in Zürich eine Vielzahl von Angeboten für junge Eltern und ihre Kinder in ganz verschiedenen Formen existiert, erschien es uns sinnvoll, eine weitere Institution zu gründen, und zwar aus folgenden Gründen:

, Es gibt selbstorganisierte Elterngruppen und Elterntreffs. Bei diesen Einrichtungen kommt es aber häufig zu Gruppenbildungen. Nicht immer fühlen sich alle an diesen Orten willkommen. Die Anwesenheit von Fachpersonen kann verhindern, dass einzelne Gruppen einen Ort für sich einnehmen und andere Eltern und Kinder ausschliessen. Insbesondere wirkt sie einer Separierung zwischen Schweizern und Westeuropäern auf der einen und aussereuropäischen und osteuropäischen Einwanderern auf der anderen Seite entgegen.

, Der Zugang ist niederschwelliger als bei einer Beratungsstelle. Man kann kommen, ohne ein Problem formulieren zu müssen, unverbindlich. Schwierige Konstellationen können sich durch die alltagsnahe Situation zeigen und von den Fachpersonen aufgenommen werden.

, Das Kind verfügt zwar noch nicht, oder kaum aktiv über die Sprache, es hat aber verschiedene Möglichkeiten der Kommunikation wie Schreien, Blickkontakte, Spiele, mit denen es etwas darstellen kann. Die Fachpersonen sind für diese Formen der «vorsprachlichen Sprache» sensibilisiert und können sie in den Diskurs aufnehmen. Die Kinder können damit aktiv an der Bearbeitung ihrer Konflikte teilnehmen, bzw. sie selbst initiieren, ohne dass sie auf die Vermittlung einer Bezugsperson angewiesen sind.

Im Zusammenhang mit dem minimalen Mutterschutz in der Schweiz besuchen immer mehr Babys schon ab dem Alter von drei Monaten die Krippe. Unter den Mitarbeitenden im Frühbereich, in Krippen, an Beratungsstellen usw. wird diskutiert, wie der Eintritt dieser Säuglinge in die Krippen gestaltet werden kann, damit er von Mutter und Kind nicht traumatisch erlebt wird. Ein Teil der an diesem Diskussionsprozess Beteiligten sind psychoanalytisch ausgebildete oder interessierte Personen, die im Austausch stehen mit der Säuglings- und Bindungs- 
forschung. Es wird darauf hin gearbeitet, dass die Ausdrucksformen des Säuglings genau beobachtet und danach gedeutet werden, ob er sich entspannt und nicht unter Stress fühlt. Erst dann sollte eine Trennung von der Mutter in Betracht gezogen werden und wenn möglich über kürzere Zeiträume. Die häufig konflikthafte Beziehung zwischen den Eltern, für die die Trennung nicht einfach ist, und der Erzieherin, die möglicherweise fürchtet, von den Eltern kritisiert zu werden, soll von einer aussenstehenden Person, z. B. der Gruppen- oder Krippenleiterin begleitet werden (von Ditfurt 2009: 64). Einige Fachpersonen fragen kritisch, ob ein so früher Eintritt in die Krippe, wenn immer möglich, nicht ganz vermieden werden sollte. In der «Maison Verte» wird versucht, die Ausdrucksformen des Säuglings, resp. Kleinkinds nicht nur aufzunehmen, sondern auch ins symbolische Register zu übersetzen. Ich vermute, dass die Lacanschen Kategorien Symbolisches - Imaginäres - Reales diesen an der Praxis orientierten Diskussionsprozess bereichern können und die «Fadenspule» auch in diesem Sinn für Zürich ein Gewinn sein kann. Die Berichte aus der psychoanalytischen Praxis von Françoise Dolto und anderen Analytikern und Analytikerinnen, sowie von Mitarbeitenden der «Maison Verte» und ähnlicher Einrichtungen zeigen eindrücklich, dass selbst ganz kleine Kinder an Orten wie der «Fadenspule» in Anwesenheit der Mutter den psychischen Prozess der Separation durchleben und eine Repräsentanz im Register des Symbolischen ausbilden können, die es ihnen ermöglicht, frühe reale Trennungen ohne übermässigen Stress zu überstehen.

\section{Literatur}

Ambass, Dagmar und Brüning, Antje (2009): «Die Fadenspule». In: UndKinder, Juni 2009. Zürich: Marie-Meierhofer-Institut für das Kind, 45-55.

Dolto, Françoise (1995): Nous irons à la maison verte. In: F. Dolto (Hrsg.). La cause des enfants. Paris: Pocket, 395-438.

Dolto, Françoise, Malandrin, Marie-Hélène und Schauder, Claude (2009): Une psychanalyste dans la cité. L'aventure de la Maison Verte. Paris: Gallimard.

Pedrina, Fernanda (2010): Babys in Gruppen. Frühe Formen des sozialen Austausches und ihr Beitrag für die kindliche Entwicklung. In: Kinderanalyse. Heft 1. Stuttgart: Klett-Cotta, 28-52.

Pikler, Emmi und Tardos, Anna (2001): Lasst mir Zeit. Die selbständige Bewegungsentwicklung des Kindes bis zum freien Gehen. Untersuchungsergebnisse, Aufsätze und Vorträge. München: Pflaum.

Saller, Vera, Würgler, Mirna (1992): FünfJahre Oase. Broschüre des Zentrum Glaubten. Zürich: Jugendamt/Sozialamt. 
Von Ditfurth, Anna (2009). «Verlust und Trauer in Übergangssituationen begleiten» in: UndKinder Nr. 83. Zürich: Marie-Meierhofer-Institut für das Kind, 57-65. Winnicott, Donald Woods (2010): Vom Spiel zur Kreativität. Stuttgart: Klett-Cotta

\section{Anmerkungen}

1 Zur «Maison Verte» siehe den Artikel von Marie Hélène Malandrin und betreffend Begegnungsorte siehe den Beitrag von Esther Leuthard in diesem Heft.

2 «Lieux d'accueil»: wörtlich «Empfangsorte»; «accueillants»: wörtlich «Empfangende», Berufsbezeichnung für die Begleitpersonen in der «Maison Verte». Uns ist kein deutscher Ausdruck eingefallen, der die Konnotation des Empfangens beinhaltet. Daher wird der französische Ausdruck beibehalten.

3 Die Fadenspule/Offener Bereich Selnau/Sihlamtsstr. 18/8003 Zürich/Öffnungszeiten: Montag und Dienstag von 9.00 bis 12.00 Uhr. Kontakt: Dagmar Ambass (Tel. 07929111 33). die.fadenspule@gmail.com und www.diefadenspule.ch (in Vorbereitung). 